\title{
Production of Scedosporium boydii CatA1 and SodC recombinant proteins: another step towards a standardized test for serodiagnosis of Scedosporium infections
}

\author{
Sara Mina a,b, Cindy Staerck a, Agnès Marot a, Charlotte Godon a, Alphonse Calenda a, \\ Jean-Philippe Bouchara a, Maxime J.J. Fleury a

\author{
a Groupe d'Etude des Interactions Hôte-Pathogène (EA 3142), UNIV Angers, UNIV Brest, Université \\ Bretagne-Loire, Angers, France \\ b Beirut Arab University, Faculty of Health Sciences, Beirut, Lebanon
}

\section{Introduction}

Scedosporium species are responsible for a wide variety of infections ranging from localized infections to disseminated infections in severely immunocompromised patients, particularly patients with cystic fibrosis (CF) undergoing lung or heart-lung transplantation. Serodiagnosis of these infections may be useful to confirm the biological diagnosis in deep-seated chronic infection, but also in CF patients with a Scedosporium-positive sputum culture to differentiate between an airway colonization and a respiratory infection. Nevertheless, it is limited to specialized laboratories using crude antigenic extracts because of the lack of commercially available serodiagnosis kits. Therefore, it may be hampered by variations from one batch of the antigenic extract to another, and cross-reactions with other pathogenic fungi including the aspergilli. To overcome these limitations, we investigated, using recombinant proteins, the antibody response in several groups of patients with or without an Aspergillus fumigatus or a Scedosporium infection, and evaluated their value as serodiagnostic tools to discriminate Aspergillus and Scedosporium infections and to differentiate between an airway colonization by Scedosporium and a respiratory infection.

\section{Production of Scedosporium recombinant proteins}

Recombinant proteins mimicking Scedosporium boydii catalase A1 (rCatA1) or cytosolic superoxide dismutase (rSodC) or $S$. apiospermum alkaline potease (rAlp1) or dipeptidylpeptidase $V$ (rDPPV) and tagged with histidine (His) or with glutathione-S-transferase (GST) were produced in Pichia pastoris or Escherichia coli. All purified recombinant proteins showed, by SDS-PAGE, a molecular mass consistent with the expected mass (100, 45, 60, 100 and $26 \mathrm{kDa}$ for rGST-SbCatA1, rGST-SbSODC, rGST-SaAlp1, rGSTSaDDPV and rGST, respectively), except rSbCatA1-(His)6 which exhibited a molecular mass of $100 \mathrm{kDa}$ instead of the $80-\mathrm{kDa}$ expected mass.

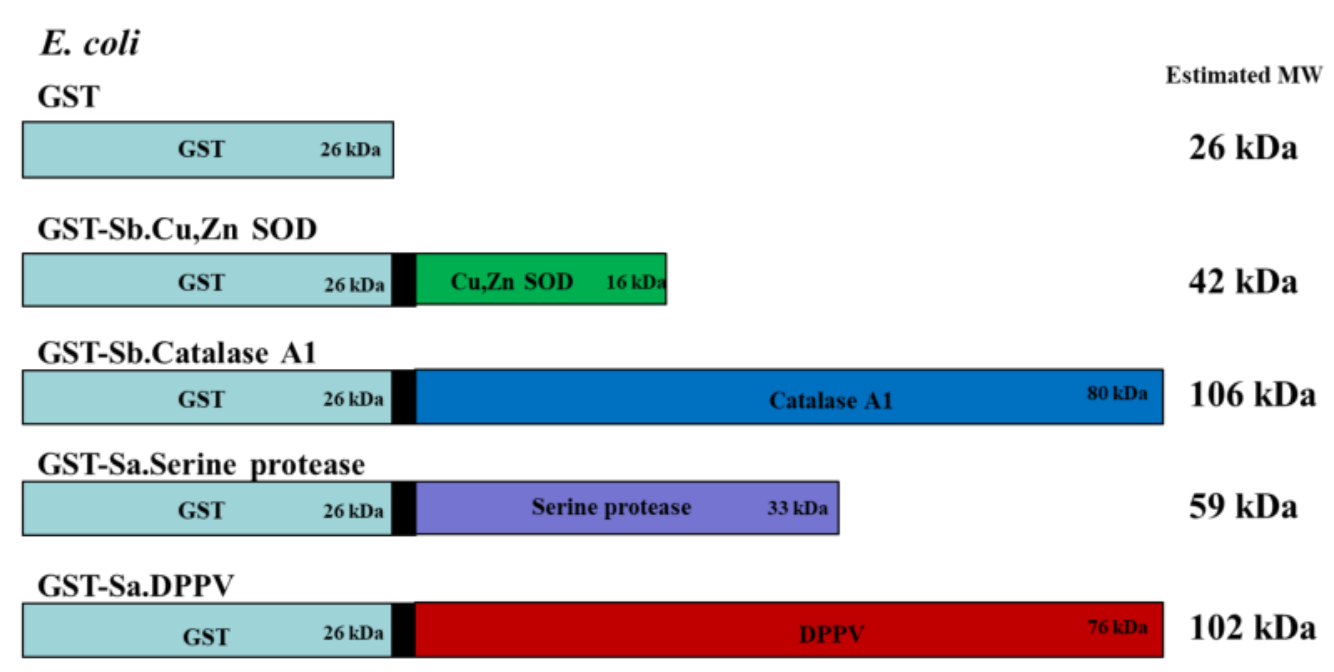

P. pastoris

Sb.Catalase A1-(His) $80 \mathrm{kDa}$

Fig. 1: Schematic representation of the recombinant proteins produced in Escherichia coli or Pichia pastoris.

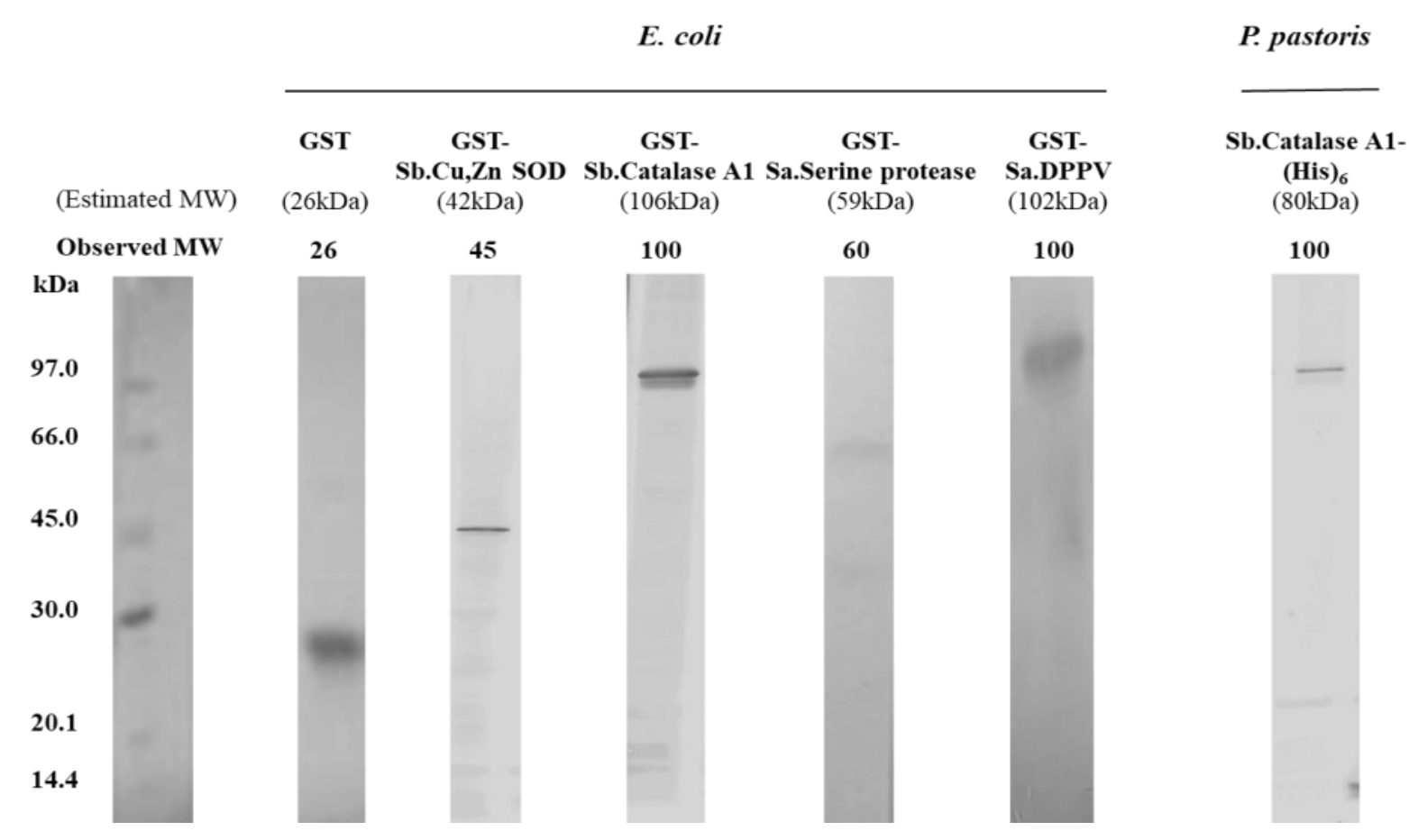

Fig. 2: SDS-PAGE analysis of the recombinant proteins produced in Escherichia coli or Pichia pastoris.

\section{Reactivity of human sera against Scedosporium recombinant proteins}

Sera from group A correspond to CF patients without any filamentous fungus recovered from sputum samples during the 6 months preceding or following blood sampling; sera from group $B$ correspond to CF patients colonized by $A$. fumigatus and with a positive antibody response exclusively against $A$. fumigatus; sera from group C correspond to CF patients colonized only by Scedosporium species, but without antibodies directed against Scedosporium species; sera from group D correspond to CF patients with an exclusive recovery of Scedosporium species from respiratory secretions and with a serological response exclusively against Scedosporium species antigenic extract. Only rCatA1 and rSodC allowed the detection of Scedosporium infection, and the differentiation with an Aspergillus infection. Combination of the results showed a sensitivity of $88.2 \%$ and a specificity of $97 \%$.

Table 1: Positivity of patient sera against rGST-SbCatA1 and rGST-SbSODC.

\begin{tabular}{llll}
\hline \multicolumn{4}{l}{ Seropositivity using } \\
\cline { 2 - 4 } & rGST-SbCatA1 & rGST-SbSODC & $\begin{array}{l}\text { Both recombinant } \\
\text { proteins }\end{array}$ \\
\hline Sensitivity & $88.2 \%$ & $88.2 \%$ & $82.4 \%$ \\
Specificity & $97 \%$ & $97.6 \%$ & $100 \%$ \\
Positive predictive value & $75 \%$ & $79 \%$ & $100 \%$ \\
Negative predictive value & $99 \%$ & $99 \%$ & $98.2 \%$ \\
\hline \multicolumn{4}{c}{ Conclusion } \\
\hline These recombinant proteins allow the differentiation between \\
Scedosporium and Aspergillus infections, as well as between \\
airway colonization and infection due to Scedosporium species, \\
which may be particularly helpful for clinicians to guide them in \\
their therapeutic decision.
\end{tabular}

